\title{
A Great Mimicker of Acute Cholecystitis in Young Population: A Case Report and Literature Review
}

\author{
Mehmet Karagülle ${ }^{1}$, Fatma Zeynep Arslan ${ }^{2 *}$, Esra Bilgi ${ }^{1}$, Süleyman Önc $\ddot{u ̈}^{1}$, Samet Şimşek ${ }^{1}$ and Mehmet \\ Öncü ${ }^{1}$
}

${ }^{1}$ Department of Radiology, Bagcılar Training and Research Hospital, University of Health Science, Konya, Turkey

${ }^{2}$ Department of Radiology, Konya Training and Research Hospital, University of Health Science, Konya, Turkey

*Corresponding author: Fatma Zeynep Arslan, Department of Radiology, Konya Training and Research Hospital, University of Health Science, Necip Fazıl Mahallesi, Fatih Cad. No:4/1, Meram, Postcode: 42090, Konya, Turkey, Tel: +905064382433, Fax: +903325121653

\begin{abstract}
Gallbladder agenesis is a rare biliary anomaly which can be important for patients complaining of chronic abdominal pain. Gallbladder agenesis can mimic cholecystitis by causing recurrent upper abdominal pain and tenderness. In a significant number of patients with gallbladder agenesis, unnecessary surgical procedures are performed because of the misdiagnosis. The morbidity of the disease can reduce, since the correct diagnosis of gallbladder agenesis decrease unnecessary surgical procedures. The diagnosis can be established with performing ultrasound and Magnetic resonance cholangiopancreatography (MRCP) examination. We reported a case of a young patient suffers from recurrent abdominal pain and diagnosed with gallbladder agenesis.
\end{abstract}

\section{Keywords}

Gallbladder agenesis, Magnetic resonance cholangiopancreatography , Biliary colic

\section{Introduction}

Gallbladder agenesis (GA) is a rare biliary anomaly which can be important for patients complaining of chronic abdominal pain. GA has a predilection for female with a reported frequency of 10 and 65 per $100,000[1,2]$. We reported a case of a young patient suffers from recurrent abdominal pain and diagnosed with GA.

\section{Case Report}

A 13-year-old female who has no known history of disease was admitted to the emergency department with right upper quadrant pain. Patient described the pain with a sudden onset, colic and radiating to the back. He described nausea, vomiting and anorexia accompanying the pain, which is aggravating with fatty foods. Laboratory tests revealed a slight increase in ALT, AST and total bilirubin values. The gallbladder was not visualized and there was no dilatation in choledochus and intrahepatic biliary tracts on abdominal ultrasound. For the initial diagnosis, contracted gall bladder and gallbladder agenesis was considered and reexamination of the gallbladder was recommended. MRCP was recommended to the patient on the second ultrasound examination performed after a fasting of 12 hours. In MRCP examination; choledochus and intrahepatic bile ducts were normal, but no gallbladder and cystic duct was seen (Figure 1). Therefore, gallbladder agenesis was diagnosed and conservative treatment was applied.

\section{Discussion}

The gallbladder and cystic canal derived from a diverticulum located in the anterior gut. The cranial portion of this diverticula forms the liver, the caudal portion creates ventral pancreas and gallbladder is originated 

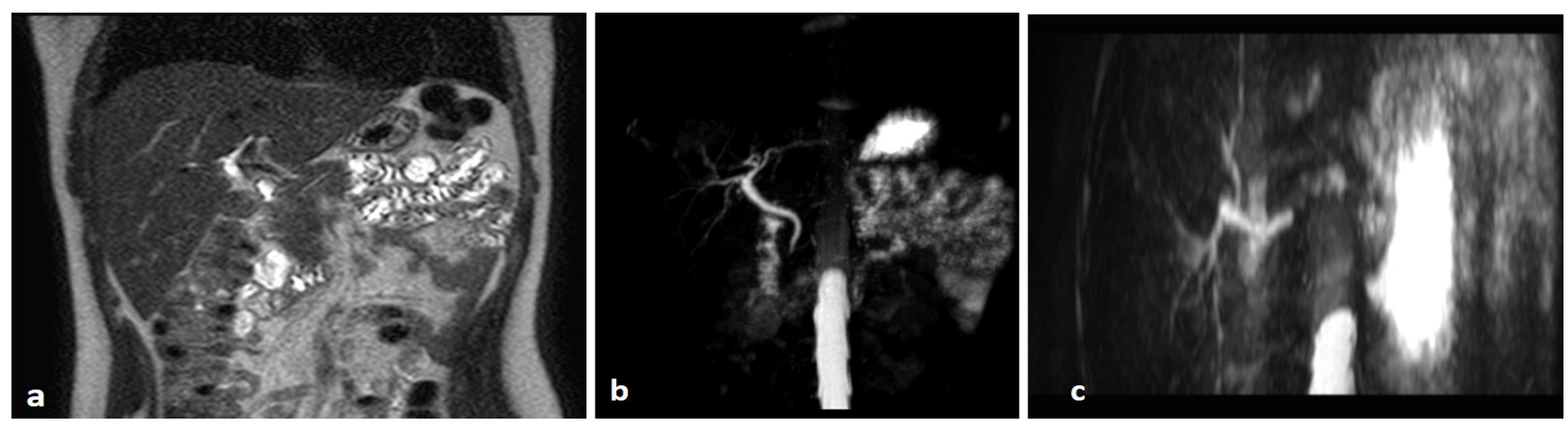

Figure 1: On T2-weighted pulse (a) Sequences and (b,c) Reformate images; choledochus and intrahepatic bile ducts were normal, but no gallbladder and cystic duct was seen.

from rest of the diverticula. GA is observed when there is a dysgenesis involving the central part of the diverticulitis. Hepatobiliary anomalies such as liver right lobe agenesis, hepatic duct atresia, quadrate lobe hypoplasia can be frequently seen as an accompanying findings [3]. Rarely, cardiac, renal, gastrointestinal, pulmonary and skeletal anomalies have been reported [3].

More than half of the patients have symptoms of biliary system until they are diagnosed in the second or third decade. Chronic recurrent abdominal pain may be associated with sphincter dysfunction [4]. Furthermore; increased choledochal stones frequency is seen in patients with gallbladder agenesis [5]. GA can mimic cholecystitis by causing recurrent upper abdominal pain and tenderness. In a significant number of patients with $G A$, unnecessary surgical procedures are performed because of the misdiagnosis [4,5]. The morbidity of the disease can reduce, since the correct diagnosis of GA decrease unnecessary surgical procedures $[6,7]$. The diagnosis can be established with performing ultrasound and MRCP examination.

In conclusion; GA can easily be overlooked by clinicians and radiologists because of their rarity in everyday practice of medicine. If gall bladder sonographically can not be visualised, wall-echo-shadow appearence or contracted gallbladder is present, cholecystitis should be reconsidered in a patient with biliary colic and GA should also be remembered when laboratory findings do not support the diagnosis of cholecystitis. MRCP examination to be performed in this case will be very useful in diagnosis.

\section{References}

1. Mittal A, Singla S, Singal R, Mehta V (2011) Gallbladder agenesis with common bile duct stone: a rare case with a brief review of the literature. Turk J Gastroenterol 22: 216218.

2. Malde S (2010) Gallbladder agenesis diagnosed intraoperatively: a case report. J Med Case Reports 4: 285.

3. Kasi PM (2011) Gallbladder agenesis. Case Reports in Gastroenterology 5: 654-662.

4. Rajkumar A, Piya A (2017) Gall bladder agenesis: a rare embryonic cause of recurrent biliary colic. Am J Case Rep 18: 334-338.

5. Seo N, Kim SY, Lee SS, Byun JH, Kim JH, et al. (2016) Sclerosing cholangitis: Clinicopathologic features, imaging Spectrum, and systemic approach to differential diagnosis. Korean J Radiol 17: 25-38.

6. Ponsioen CY (2015) Diagnosis, differential diagnosis, and epidemiology of primary Sclerosing cholangitis. Dig Dis 33: 134-139.

7. Yam BL, Siegelman ES (2014) MR imaging of the biliary system. Radiol Clin N Am 52: 725-755. 